\title{
Matrices with restricted entries and $q$-analogues of permutations (extended abstract)
}

\author{
Joel Brewster Lewis ${ }^{1}$, Ricky Ini Liu ${ }^{2}$, Alejandro H. Morales ${ }^{1}$, Greta Panova ${ }^{3}$, \\ Steven V Sam ${ }^{1}$ and Yan Zhang ${ }^{1}$ \\ ${ }^{1}$ Department of Mathematics, Massachusetts Institute of Technology, Cambridge, MA, USA \\ ${ }^{2}$ School of Mathematics, University of Minnesota, Minneapolis, MN, USA \\ ${ }^{3}$ Department of Mathematics, Harvard University, Cambridge, MA, USA
}

\begin{abstract}
We study the functions that count matrices of given rank over a finite field with specified positions equal to zero. We show that these matrices are $q$-analogues of permutations with certain restricted values. We obtain a simple closed formula for the number of invertible matrices with zero diagonal, a $q$-analogue of derangements, and a curious relationship between invertible skew-symmetric matrices and invertible symmetric matrices with zero diagonal. In addition, we provide recursions to enumerate matrices and symmetric matrices with zero diagonal by rank. Finally, we provide a brief exposition of polynomiality results for enumeration questions related to those mentioned, and give several open questions.

Résumé. Nous étudions certaines fonctions qui comptent des matrices à coefficients dans un corps fini d'un rang donné ayant certaines entrées égales à zéro. Nous montrons que ces matrices sont des $q$-analogues des permutations avec certaines valeurs restreintes, et nous obtenons une formule simple et fermée pour calculer le nombre de matrices inversibles avec zéro sur toute la diagonale. De plus nous donnons des récursions pour énumérer par le rang les matrices et les matrices symétriques avec des zéros sur la diagonale. Pour finir, nous faisons un exposé concis des résultats sur la polynomialité des fonctions énumératives liées à celles qui sont mentionnées antérieurement, et nous incluons plusieurs questions ouvertes.

Resumen. Estudiamos ciertas funciones que cuentan matrices con un rango dado sobre un campo finito y con ciertas entradas iguales a cero. Mostramos que estas matrices son un $q$-análogo de permutaciones con ciertos valores restringidos. También obtenemos una recursión simple y cerrada para el número de matrices invertibles con ceros en toda la diagonal. Además, damos recursiones para enumerar matrices y matrices simétricas con ceros en la diagonal por rango. Finálmente, damos una exposición breve de resultados sobre la polinomialidad de funciones enumerativas relacionadas a las anteriormente mencionadas e incluimos varias preguntas abiertas.
\end{abstract}

Keywords: linear algebra over finite fields, $q$-analogues, derangements

\section{Introduction}

Fix a prime power $q$. Let $\mathbf{F}_{q}$ denote the field with $q$ elements and let $\mathbf{G L}(n, q)$ denote the group of $n \times n$ invertible matrices over $\mathbf{F}_{q}$. The support of a matrix $\left(A_{i j}\right)$ is the set of indices $(i, j)$ such that $A_{i j} \neq 0$. 
Our work was initially motivated by the following question of Richard Stanley: how many matrices in $\mathbf{G L}(n, q)$ have support avoiding the diagonal entries? The answer to this question is

$$
q^{\left(\begin{array}{c}
n-1 \\
2
\end{array}\right)-1}(q-1)^{n}\left(\sum_{i=0}^{n}(-1)^{i}\left(\begin{array}{c}
n \\
i
\end{array}\right)[n-i]_{q} !\right),
$$

which is proven in Proposition 2.1 as part of a more general result. This question has a natural combinatorial appeal and is reminiscent of the work of Buckheister [Buc] and Bender [Ben] enumerating invertible matrices over $\mathbf{F}_{q}$ with trace zero (see also [Sta1, Prop. 1.10.15]). It also falls naturally into two broader contexts, the study of $q$-analogues of permutations and the study of polynomiality results for certain counting problems related to algebraic varieties over $\mathbf{F}_{q}$.

In the former context, we consider the following situation: fix $m, n \geq 1, r \geq 0$, and $S \subset\{(i, j) \mid 1 \leq$ $i \leq m, 1 \leq j \leq n\}$. Let $T_{q}$ be the set of $m \times n$ matrices $A$ over $\mathbf{F}_{q}$ with rank $r$ and support contained in the complement of $S$. Also, let $T_{1}$ be the set of 0-1 matrices with exactly $r$ 1's, no two of which lie in the same row or column, and with support contained in the complement of $S$ (i.e., the set of rook placements avoiding $S$ ). We have that $T_{1}$ is a $q$-analogue of $T_{q}$, in the following precise sense:

Proposition 5.1 We have $\# T_{q} \equiv \# T_{1} \cdot(q-1)^{r}\left(\bmod (q-1)^{r+1}\right)$.

In particular, when $\# T_{q}$ is a polynomial function of $q$ we have that $\# T_{q}$ is divisible by $(q-1)^{r}$ and $\# T_{q} /\left.(q-1)^{r}\right|_{q=1}=\# T_{1}$. Thus, rank $r$ matrices whose support avoids the set $S$ can be seen as a $q$ analogue of rook placements that avoid $S$. Applying this to our situation where $S$ is the set of diagonal entries, we get that the set of invertible matrices avoiding the diagonal is a $q$-analogue of the set of derangements, a fact that can also be seen directly from the explicit formula above. (There is also a more conceptual explanation for this using the Bruhat decomposition of GL $(n, q)$ : see [LLMPSZ, Sec. 2.2].)

Note that for an arbitrary set $S$ of positions, the function $\# T_{q}$ need not be a polynomial in $q$. (Stembridge [Ste1] gives an example of non-polynomial $\# T_{q}$ with $n=m=7, r=7$, and a set $S$ with $\# S=28$.) The second context concerns the question of which sets $S$ give a polynomial $\# T_{q}$ and is deeply related to a speculation of Kontsevich from 1997 (see Stanley [Sta2] and Stembridge [Ste1]) that was proven false by Belkale and Brosnan $[\mathrm{BB}]$. We provide further background on this topic in Section 5

We close this introduction with a summary of the results of our paper.

Section 2 is concerned with Stanley's question on the enumeration of matrices in $\mathbf{G L}(n, q)$ with zero diagonal. We attack this problem by enumerating larger classes of matrices. We provide two recursions, one based on the size of the matrix and the other based on the rank of the matrix, and we provide a closed-form solution for the first recursion.

In Section 3, we enumerate symmetric matrices in $\mathbf{G L}(n, q)$ whose support avoids the diagonal in the case that $n$ is even. These matrices may be viewed as a $q$-analogue of fixed point-free involutions. A curious byproduct of our formula (originally due to Jones [Jon]) is that it also counts the number of symmetric matrices in $\mathbf{G L}(n-1, q)$ and the number of skew-symmetric matrices in $\mathbf{G} \mathbf{L}(n, q)$. In fact, the varieties associated to these three classes of matrices are pairwise non-isomorphic, and we have not found a satisfactory reason that their solution sets have the same size.

In Section 4, we attack the general problem of enumerating symmetric matrices with zeroes on the diagonal with given rank. We provide recursions for arbitrary rank and solve the full rank case to obtain the enumeration of symmetric matrices in $\mathbf{G L}(n, q)$ when $n$ is odd. The situation in this case is significantly more complicated than in Sections 2 and 3 
Finally, in Section 5 we revisit Proposition 5.1, discuss the two broader contexts mentioned above, and give some open questions about families of sets $S$ for which $\# T_{q}(m \times n, S, r)$ is a polynomial in $q$.

For a complete version of this extended abstract, see [LLMPSZ].

\section{Notation}

Given an integer $n$, we define the $q$-number $[n]_{q}=\frac{q^{n}-1}{q-1}$, the $q$-factorial $[n]_{q} !=[n]_{q} \cdot[n-1]_{q} \cdot[n-2]_{q} \cdots$ and the $q$-double factorial $[n]_{q} ! !=[n]_{q} \cdot[n-2]_{q} \cdot[n-4]_{q} \cdots$. In addition, we use a number of invented notations; to avoid confusion and for easy reference, we include a table of these functions here. The last column indicates the sections in which the notation is used.

\begin{tabular}{|c|c|c|c|c|}
\hline set & \# set & description & & ectio \\
\hline $\operatorname{Mat}_{0}(n, k, r)$ & $\operatorname{mat}_{0}(n, k, r)$ & $\begin{array}{l}\text { set of } n \times n \text { matrices of rank } r \text { over } \mathbf{F}_{q} \text { with first } k \\
\text { diagonal entries equal to zero }\end{array}$ & 2 & \\
\hline $\operatorname{Sym}(n)$ & $\operatorname{sym}(n)$ & set of $n \times n$ symmetric invertible matrices over $\mathbf{F}_{q}$ & & 4 \\
\hline $\operatorname{Sym}(n, r)$ & $\operatorname{sym}(n, r)$ & set of $n \times n$ symmetric matrices over $\mathbf{F}_{q}$ of rank $r$ & 4 & \\
\hline $\operatorname{Sym}_{0}(n, r)$ & $\operatorname{sym}_{0}(n, r)$ & $\begin{array}{l}\text { set of } n \times n \text { symmetric matrices with rank } r \text { over } \\
\mathbf{F}_{q} \text { with diagonal entries equal to zero }\end{array}$ & 司 & \\
\hline $\mathrm{S}(n, k)$ & $\mathrm{s}(n, k)$ & $\begin{array}{l}\text { set of } n \times n \text { symmetric invertible matrices with first } \\
k \text { diagonal entries equal to zero }\end{array}$ & & 4 \\
\hline $\operatorname{Sym}_{0}(n, k, r)$ & $\operatorname{sym}_{0}(n, k, r)$ & $\begin{array}{l}\text { set of } n \times n \text { symmetric matrices with rank } r \text { with } \\
\text { first } k \text { diagonal entries equal to zero }\end{array}$ & 14 & \\
\hline$T_{q}(m \times n, S, r)$ & $\# T_{q}(m \times n, S, r)$ & $\begin{array}{l}\text { set of } m \times n \text { matrices over } \mathbf{F}_{q} \text { with rank } r \text { and sup- } \\
\text { port contained in the complement of } S\end{array}$ & & 5 \\
\hline$T_{1}(m \times n, S, r)$ & $\# T_{1}(m \times n, S, r)$ & $\begin{array}{l}\text { set of } 0-1 \text { matrices with exactly } r 1 \text { 's, no two of } \\
\text { which lie in the same row or column, and with sup- } \\
\text { port contained in the complement of } S\end{array}$ & & . \\
\hline
\end{tabular}

\section{Matrices with zeroes on the diagonal}

In this section, we consider the problem of counting invertible matrices over $\mathbf{F}_{q}$ with zero diagonal. In Section 2.1 we recursively count full rank matrices of rectangular shape with all-zero diagonal and in Section 2.2 we recursively count square matrices by rank and number of zeroes on the diagonal. We solve the first recursion and obtain a closed form formula for the number of invertible matrices with zeroes on the diagonal. These numbers give an enumerative $q$-analogue of the derangements, i.e., dividing all factors of $q-1$ and setting $q=1$ in the result gives the number of derangements.

\subsection{Recursion by size}

For $1 \leq k \leq n$, denote by $f_{k, n}$ the number of $k \times n$ matrices $A$ over $\mathbf{F}_{q}$ such that $A$ has full rank $k$ and such that $A_{i i}=0$ for $1 \leq i \leq k$. We give a recursive proof of a simple explicit formula for $f_{k, n}$. In particular, we will have a formula for $f_{n, n}$, the number of invertible $n \times n$ matrices with zeroes on the diagonal. 
648 Joel Brewster Lewis, Ricky Ini Liu, Alejandro H. Morales, Greta Panova, Steven V Sam, Yan Zhang

Proposition 2.1 For $1 \leq k \leq n$,

$$
f_{k, n}=q^{\left(\begin{array}{c}
k-1 \\
2
\end{array}\right)}(q-1)^{k}\left(q^{-1} \sum_{i=0}^{k}(-1)^{i}\left(\begin{array}{c}
k \\
i
\end{array}\right) \frac{[n-i]_{q} !}{[n-k]_{q} !}\right)
$$

is the number of $k \times n$ matrices of rank $k$ with zeroes on the diagonal. In particular,

$$
f_{n, n}=q^{\left(\begin{array}{c}
n-1 \\
2
\end{array}\right)}(q-1)^{n}\left(q^{-1} \sum_{i=0}^{n}(-1)^{i}\left(\begin{array}{c}
n \\
i
\end{array}\right)[n-i]_{q} !\right)
$$

is the number of invertible $n \times n$ matrices with zeroes on the diagonal.

Proof idea: We proceed recursively, building matrices up by adding one row at a time. This leads to the recursion

$$
f_{k+1, n}=q^{k-1}(q-1)\left(f_{k, n} \cdot[n-k]_{q}-f_{k, n-1}\right)
$$

with initial values $f_{1, n}=q^{n-1}-1$, from which the result follows.

Remark 2.2 In the expression for $f_{n, n}$, the $q=1$ specialization of the alternating sum is

$$
\sum_{i=0}^{n}(-1)^{i}\left(\begin{array}{c}
n \\
i
\end{array}\right)(n-i) !=n ! \sum_{i=0}^{n} \frac{(-1)^{i}}{i !}
$$

which is the number of derangements of length $n$. The above proof does not "explain" this fact, but one can give more conceptual proofs based on the Bruhat decomposition of $\mathbf{G L}(n, q)$ (see [LLMPSZ Sec. 2.2] for details) or on Proposition 5.1

\subsection{Recursion by rank}

In this section, we use recursive methods to attack the problem of enumerating square matrices with a prescribed number of zeroes on the diagonal by rank. We use the following strategy: each $n \times n$ matrix can be inflated to $q^{2 n+1}$ different $(n+1) \times(n+1)$ matrices, and we count these by keeping careful track of what their rank is and how many zeroes they have on the diagonal. We are unable to obtain a closed formula for this recursion, but the we use the same proof technique successfuly in Sections 3 and 4.

Let $\operatorname{mat}_{0}(n, k, r)$ be the number of $n \times n$ matrices over $\mathbf{F}_{q}$ of rank $r$ whose first $k$ diagonal entries are zero (and the other diagonal entries may or may not be zero).

Proposition 2.3 We have the following recursion:

$\operatorname{mat}_{0}(n+1, k+1, r+1)=\frac{1}{q} \operatorname{mat}_{0}(n+1, k, r+1)+\left(q^{r+1}-q^{r}\right) \operatorname{mat}_{0}(n, k, r+1)-\left(q^{r}-q^{r-1}\right) \operatorname{mat}_{0}(n, k, r)$

with initial conditions

$$
\operatorname{mat}_{0}(n, 0, r)=\frac{q^{\left(\begin{array}{c}
r \\
2
\end{array}\right)}(q-1)^{r}}{[r]_{q} !}\left(\prod_{i=0}^{r-1}[n-i]_{q}\right)^{2} .
$$


Proof idea: We proceed by decomposing each $(n+1) \times(n+1)$ matrix $A$ as $A=\left[\begin{array}{ll}a & u \\ v & B\end{array}\right]$ where $a$ is an element of $\mathbf{F}_{q}, u$ is a row vector over $\mathbf{F}_{q}$ of length $n$, and $v$ is a column vector over $\mathbf{F}_{q}$ of length $n$, and $B$ is an $n \times n$ matrix. We compute for each $B$ the possible ranks of $A$, taking into account whether $a=0$ and sum over all $B$ to get the recursion.

The preceding recursion works by reducing the number of zeroes required to lie on the diagonal. However, we can easily modify the proof to work only with matrices of all-zero diagonal.

Corollary 2.4 For $r \geq 0$, the number $g_{n, r}$ of $n \times n$ matrices over $\mathbf{F}_{q}$ of rank $r$ and with zero diagonal satisfies the recursion

$$
\begin{aligned}
g_{n+1, r+1}=\left(q^{n}-q^{r-1}\right)^{2} g_{n, r-1}+\left(q^{2 r+1}+q^{r+1}-q^{r}\right) & g_{n, r+1} \\
& +\left(2 q^{n+r}-q^{2 r}-q^{2 r-1}-q^{r}+q^{r-1}\right) g_{n, r}
\end{aligned}
$$

with initial conditions $g_{n, 0}=1, g_{n,-1}=0$ and $g_{1,1}=0$.

\section{Symmetric and skew-symmetric matrices}

A natural next step is to consider symmetric matrices, which are (at least morally) a $q$-analogue of involutions, suggesting the possibility of interesting combinatorial results. This also brings us closer to a speculation by Kontsevich (see Section 5). In this section, we begin by enumerating symmetric invertible matrices over $\mathbf{F}_{q}$ whose diagonal is all zero, a $q$-analogue of involutions with no fixed points. This leads to two very unintuitive facts: in Section 3.1, we show that the number of these matrices of size $2 n$ is the same as the number of invertible symmetric matrices of size $2 n-1$; in Section 3.2, we show that both of these numbers are equal to the number of invertible skew-symmetric matrices of the size $2 n$. We give more refined but less beautiful versions of the first of these results in Section 4.

While extending the approach of Section 2.1 to the case of symmetric matrices seems impossible, the ideas of Section 2.2 can be adjusted to work in this context. The major complicating factor is that the bilinear form $u B v$ that we worked with implicitly in Section 2.2 must be replaced with the quadratic form $v B v^{T}$. Quadratic forms behave very differently in even and odd characteristic, so we give the following proviso:

Remark 3.1 Our proofs of the results in this section are only valid for $q$ odd.

Of course, some of the results still hold when $q$ is even: for example, symmetric matrices of rank $r$ with all-zero diagonal are equinumerous with skew-symmetric matrices of rank $r$ over fields of characteristic 2 for the silly reason that they are exactly the same set of matrices. For a more thorough treatment of the case $q$ even, see [Mac] and [Sta2].

\subsection{Symmetric matrices with zeroes on the diagonal}

Let $\operatorname{Sym}_{0}(n)$ denote the set of $n \times n$ symmetric matrices in $\mathbf{G L}(n, q)$ with zero diagonal and let $\operatorname{sym}_{0}(n)=$ \# $\operatorname{Sym}_{0}(n)$. Similarly, let $\mathrm{S}(n, k)$ be the set of $n \times n$ symmetric matrices in $\mathbf{G L}(n, q)$ whose first $k$ diagonal entries are zero and let $\mathrm{s}(n, k)=\# \operatorname{Sym}_{0}(n, k)$, so $\mathrm{S}(n, n)=\operatorname{Sym}_{0}(n)$.

In [Mac, Theorem 2], MacWilliams shows 
Theorem The number of symmetric invertible matrices (for any characteristic) is

$$
\operatorname{sym}(n)=q^{\left(\begin{array}{c}
n+1 \\
2
\end{array}\right)} \prod_{j=1}^{\lceil n / 2\rceil}\left(1-q^{1-2 j}\right) .
$$

Observe that when $n$ is even, $\operatorname{sym}(n-1)$ is a $q$-analogue of $(n-1) !$, the number of fixed point-free involutions in $\mathfrak{S}_{n}$.

Theorem 3.3 When $n$ is even, the number of $(n-1) \times(n-1)$ symmetric invertible matrices is equal to the number of $n \times n$ symmetric invertible matrices with zero diagonal, i.e., $\operatorname{sym}(n-1)=\operatorname{sym}_{0}(n)$.

Proof idea: Naively, one might expect that about $q^{-k}$ of all matrices in $\operatorname{Sym}(n)$ have first $k$ diagonal entries equal to 0 . We begin by showing that, remarkably, this estimate is actually exact when $n$ is even. The proof of this lemma proceeds by decomposing a matrix as in Section 2.2 followed by the classification of symmetric bilinear forms over fields of odd characteristic (see [Wan, Theorem 1.22]). Thanks to this lemma and Equation [3.2], the proof is an easy induction.

Note that the case for $q$ even was done by MacWilliams [Mac, Theorems 2, 3] (see also Equation (4.3)).

\subsection{Skew-symmetric matrices}

In this section, we count invertible skew-symmetric matrices by rank, obtaining a $q$-analogue of fixed point-free "partial involutions." It is not clear a priori that there is any connection between these matrices and symmetric matrices, but we obtain as a consequence of the result that the when $n$ is even, the number of $n \times n$ invertible skew-symmetric matrices is the same as the number of $(n-1) \times(n-1)$ invertible symmetric matrices (and so, by Theorem 3.3 , also the same as the number of $n \times n$ invertible symmetric matrices with all-zero diagonal). After the first write-up of this paper we found that this was proven by Jones [Jon, Theorems 1.7, 1.7', 1.8', 1.9] using topological methods.

Proposition 3.4 Let $\operatorname{sk}(n, r)$ be the number of $n \times n$ skew-symmetric matrices of rank $r$. When $r$ is odd we have $\operatorname{sk}(n, r)=0$ and when $r$ is even we have

$$
\operatorname{sk}(n, r)=q^{r(r-2) / 4}(1-q)^{r / 2} \cdot \frac{[n]_{q} !}{[n-r]_{q} ! \cdot[r]_{q} ! !} .
$$

In particular, when $r=n$ is even we have $\operatorname{sk}(n, n)=\operatorname{sym}(n-1)$.

One interesting observation is that this is a $q$-analogue of $\left(\begin{array}{l}n \\ r\end{array}\right)(r-1)$ !!, the number of "partial involutions of rank $r$ " with no fixed points, i.e., the number of pairs of an $r$-subset of $\{1, \ldots, n\}$ together with a fixed point-free involution on that set. Note that in [LLMPSZ, Sec. 3.3] we give another proof of $\operatorname{sk}(n, n)=\operatorname{sym}(n-1)$ via Schubert varieties.

Proof idea: We decompose the skew-symmetric matrices in the same way as in preceding sections to obtain the recurrence

$$
\operatorname{sk}(n, r)=q^{r} \operatorname{sk}(n-1, r)+\left(q^{n-1}-q^{r-2}\right) \operatorname{sk}(n-1, r-2),
$$

with initial values $\operatorname{sk}(n, 0)=1$ and $\operatorname{sk}(n, 1)=0$, whose solution is the formula stated above. In the case $r=n$ is even, compare with Equation (3.2) to obtain $\operatorname{sk}(n, n)=\operatorname{sym}(n-1)$. 


\section{Refined enumeration of symmetric matrices}

In this section, we attack the problem of enumerating $n \times n$ symmetric matrices over $\mathbf{F}_{q}$ with zeroes on the diagonal by rank. Roughly speaking, we should expect this problem to be a $q$-analogue of counting fixed point-free involutions, or of "partial fixed point-free involutions" when we consider matrices of less than full rank. As in the preceding sections, we construct a recursion to count the desired objects. Our basic approach is the same as in Section 2.2. The main difference is that the symmetry of our matrices forces us to introduce a sort of parity condition depending on whether or not we can write a matrix in the form $M \cdot M^{T}$ for some other matrix $M$. The details on whether or not we can do this are different for odd and even characteristic. We begin by mentioning both cases to then restrict our attention and results to the odd case.

Remark 4.1 ( $q$ even) It was shown by Albert [Alb. Thm. 7] that a symmetric matrix $A$ in $\mathbf{G L}(n, q)$ can be factored in the form $A=M \cdot M^{T}$ for some matrix $M$ in $\mathbf{G L}(n, q)$ if and only if $A$ has at least one nonzero diagonal entry. Thus $\#\left\{A \in \operatorname{Sym}(n) \mid A=M \cdot M^{T}\right.$ for some $\left.M \in \mathbf{G L}(n, q)\right\}=\operatorname{sym}(n)-\operatorname{sym}_{0}(n)$. MacWilliams [Mac] gave an elementary proof of Albert's theorem and also calculated $\operatorname{sym}_{0}(n, r)$, the number of $n \times n$ symmetric matrices of rank $r$ with zero diagonal, when $q$ is even.

Theorem [Mac, Thm. 3, Sec. III] For q even, if $r=2 s+1$ is odd then

$$
\operatorname{sym}_{0}(n, 2 s+1)=0
$$

while if $r=2 s$ is even then

$$
\operatorname{sym}_{0}(n, 2 s)=\prod_{i=1}^{s} \frac{q^{2 i-2}}{q^{2 i}-1} \prod_{i=0}^{2 s-1}\left(q^{n-i}-1\right) .
$$

Henceforth, we will always assume that $q$ is odd.

For $q$ odd, define $\psi: \mathbf{F}_{q}^{\times} \rightarrow\{+,-\}$ by $\psi(\delta)=+$ if and only if $\delta$ is a perfect square in $\mathbf{F}_{q}$. In other words, $\psi$ is the Legendre symbol for $\mathbf{F}_{q}$. We can also extend $\psi$ naturally to symmetric matrices using the following remark.

Remark 4.4 By applying symmetric row and column reductions, every $n \times n$ symmetric matrix $A$ of rank $r>0$ can be written either in the form $A=M \cdot \operatorname{diag}\left(1^{r}, 0^{n-r}\right) \cdot M^{T}$ for some $M \in \mathbf{G L}(n, q)$ or in the form $M \cdot \operatorname{diag}\left(1^{r-1}, z, 0^{n-r}\right) \cdot M^{T}$ for some non-perfect square $z \in \mathbf{F}_{q}$ and some $M \in \mathbf{G} \mathbf{L}(n, q)$.

In the former case we say that $A$ has (quadratic) character $\psi(A)=\psi(1)=+$ and in the latter case we say it has character $\psi(A)=\psi(z)=-$. Two notable special cases are that if $A \in \mathbf{G L}(n, q)$ then $\psi(A)=\psi(\operatorname{det} A)$, while if $A$ is diagonal then $\psi(A)=+$ if and only if the product of the nonzero diagonal entries of $A$ is a square in $\mathbf{F}_{q}$.

Let $\operatorname{sym}_{0}(n, k, r)$ be the number of $n \times n$ symmetric matrices with rank $r$ and the first $k$ diagonal elements equal to 0 , with no other restrictions. Thus, we have for example that $\operatorname{sym}_{0}(n, n, r)=\operatorname{sym}_{0}(n, r)$ while $\operatorname{sym}_{0}(n, 0, r)=\operatorname{sym}(n, r)$ is the number of symmetric matrices of rank $r$ with no other restrictions which has been calculated in [Mac, Thm. 2, Sect. III]. Let $\operatorname{sym}_{0}^{\psi}(n, r, k)$ count only those matrices that have character $\psi$. We now give a recurrence for $\operatorname{sym}_{0}(n, k, r)$. We use this recurrence to enumerate invertible symmetric matrices over $\mathbf{F}_{q}$ with zero diagonal (Theorem 4.7), generalizing Theorem 3.3 . 
Proposition 4.5 If $r$ is odd, define $t=0$ if $(-1)^{(r+1) / 2}$ is a square in $\mathbf{F}_{q}$ and $t=1$ otherwise. Then

$$
\begin{aligned}
\operatorname{sym}_{0}^{\psi}(n+1, k+1, & r+1)=\frac{1}{q} \operatorname{sym}_{0}^{\psi}(n+1, k, r+1)+ \\
& +(-1)^{t} \cdot \psi \cdot\left(\frac{1}{2} \operatorname{sym}_{0}(n, k, r)+\operatorname{sym}_{0}^{\psi}(n, k, r+1)\right) \cdot\left(q^{(r+1) / 2}-q^{(r-1) / 2}\right) .
\end{aligned}
$$

If $r$ is even and $r>0$, define $t=0$ if $(-1)^{r / 2}$ is a square in $\mathbf{F}_{q}$ and $t=1$ otherwise. Then

$$
\begin{aligned}
\operatorname{sym}_{0}^{\psi}(n+1, k+1, r+1)=\frac{1}{q} \operatorname{sym}_{0}^{\psi}( & n+1, k, r+1)- \\
& -\frac{(-1)^{t}}{2}\left(\operatorname{sym}_{0}^{+}(n, k, r)-\operatorname{sym}_{0}^{-}(n, k, r)\right)\left(q^{r / 2}-q^{r / 2-1}\right) .
\end{aligned}
$$

We have initial values

$$
\begin{gathered}
\operatorname{sym}_{0}^{\psi}(n+1, k+1,1)=\frac{1}{2} \operatorname{sym}_{0}(n+1, k+1,1)=\frac{q-1}{2} \sum_{i=0}^{n-k-1} q^{i}=\frac{q^{n-k}-1}{2}, \\
\operatorname{sym}_{0}^{+}(n, 0,2 s+1)=\frac{1}{2} \operatorname{sym}(n, 2 s+1)
\end{gathered}
$$

and

$$
\operatorname{sym}_{0}^{+}(n, 0,2 s)=\frac{1}{2} \frac{q^{s}+(\psi(-1))^{s}}{q^{s}} \operatorname{sym}(n, 2 s) .
$$

Proof idea: As before, we proceed by building larger matrices by adding rows and columns to smaller matrices; for each $n \times n$ symmetric matrix $B$, we consider the $(n+1) \times(n+1)$ matrices of the form

$$
A=\left[\begin{array}{cc}
a & v \\
v^{T} & B
\end{array}\right]
$$

and analyze them (taking into account whether $a=0$ ) to write down a recursion. The number of matrices $A$ of a given rank associated to a matrix $B$ now depends on the rank of $B$ (as in Proposition 2.3) and also on its quadratic character. At important junctures we use Wan's result [Wan, Theorem 1.26] on the number of zeroes over $\mathbf{F}_{q}$ of certain quadratic forms. The $k=0$ base cases are provided by [Mac, Thm. 2, Sect. III].

We do not have a solution for this recurrence. However, we use it to obtain two partial results towards its solution:

Corollary 4.6 We have

$$
\operatorname{sym}_{0}^{+}(n+1,, k+1,2 s+1)=\operatorname{sym}_{0}^{-}(n+1, k+1,2 s+1)=\frac{1}{2} \operatorname{sym}_{0}(n+1, k+1,2 s+1),
$$

and

$\operatorname{sym}_{0}(n+1, k+1,2 s)+\operatorname{sym}_{0}(n+1, k+1,2 s+1)=\frac{1}{q^{k+1}}(\operatorname{sym}(n+1,2 s)+\operatorname{sym}(n+1,2 s+1))$. 
We also use Proposition 4.5 to obtain an explicit formula in the case of invertible symmetric matrices (i.e., when $r=n$ ). Let $\mathrm{s}(n, k)=\operatorname{sym}_{0}(n, k, n)$ be the number of invertible $n \times n$ symmetric matrices with first $k$ diagonal elements are equal to 0 , with no other restrictions. We use the recurrence in Proposition 4.5 to give a recurrence for this full rank case.

Theorem 4.7 Let $\mathrm{s}(n, k)$ be the number of invertible $n \times n$ symmetric matrices with the first $k$ diagonal elements equal to 0 and let $\operatorname{sym}(n)$ be the number of invertible $n \times n$ symmetric matrices with no other restrictions. We have

$$
\mathrm{s}(2 m, k+1)=\frac{1}{q^{k+1}} \operatorname{sym}(2 m)
$$

and

$$
\mathrm{s}(2 m+1, k+1)=\frac{1}{q^{k+1}} \sum_{j=0}^{2 m+1}(-1)^{\lceil j / 2\rceil} q^{\lfloor j / 2\rfloor(2 m+1-\lfloor j / 2\rfloor)}(q-1)^{j}\left(\begin{array}{c}
k+1 \\
j
\end{array}\right) \operatorname{sym}(2 m+1-j) .
$$

Proof idea: Substitute $r=n$ into Proposition 4.5, sum up the two different characters, and iterate.

\section{Polynomiality, $q$-analogues, and some open questions}

So far, we have fixed sets of the form $S=\{(i, i) \mid 1 \leq i \leq k\}$, counted matrices over $\mathbf{F}_{q}$ with support avoiding $S$ by rank, and done analogous counts for symmetric and skew-symmetric matrices. In this section, we briefly examine what happens when we enumerate matrices of given rank whose support avoids an arbitrary fixed set of entries.

\section{$5.1 q$-analogues and the proof idea of Proposition 5.1}

Fix $m, n \geq 1, r \geq 0$, and $S \subset\{(i, j) \mid 1 \leq i \leq m, 1 \leq j \leq n\}$. Let $T_{q}=T_{q}(m \times n, S, r)$ be the set of $m \times n$ matrices $A$ over $\mathbf{F}_{q}$ with rank $r$ and support contained in the complement of $S$. We consider the problem of computing $\# T_{q}$, the number of such matrices.

A first observation is that, holding $m, n, r, S$ fixed and letting $q$ vary, the function $\# T_{q}$ need not be polynomial in $q$. We have already seen this phenomenon in the case of symmetric matrices; for instance, setting $m=n=r$ to be an odd positive integer and $S=\{(i, i) \mid 1 \leq i \leq n\}$ we have from Equations (4.2) and 4.3) and Theorem 4.7 that $\#\left(T_{q}(n \times n, S, n) \cap \operatorname{Sym}(n)\right)=\operatorname{sym}_{0}(n)$ is equal to zero when $q$ is even but is nonzero when $q$ is odd. This lack of polynomiality also occurs in the not-necessarily symmetric case. Stembridge [Ste1, Section 7] showed that for $n=m=7$, if $S^{\prime}$ is the complement of the incidence matrix of the Fano plane, then the number of invertible $7 \times 7$ matrices in $\mathbf{F}_{q}$ whose support avoids $S^{\prime}$ is given by two different polynomials depending on whether $q$ is even or odd. (This is the smallest such example in the sense that $T_{q}(n \times n, S, n)$ is a polynomial if $n<7$ for any set $S$, and if $n=7$ and $\# S>28$.)

A second observation is that we expect $\# T_{q}$ to be a $q$-analogue of a closely related problem for permutations. Specifically, let $T_{1}=T_{1}(m \times n, S, r)$ be the set of 0 -1 matrices with exactly $r$ 's, no two of which lie in the same row or column, and with support contained in the complement of $S$. The following proposition makes this precise. 
Proposition 5.1 Fix $m, n \geq 1, r \geq 0$, and $S \subset\{(i, j) \mid 1 \leq i \leq m, 1 \leq j \leq n\}$. Let $T_{q}=$ $T_{q}(m \times n, S, r)$ be the set of $m \times n$ matrices $A$ over $\mathbf{F}_{q}$ with rank $r$ and support contained in the complement of $S$, and $T_{1}$ be the set of 0-1 matrices with exactly $r$ 1's, no two of which lie in the same row or column, and with support contained in the complement of $S$. Then we have

$$
\# T_{q} \equiv \# T_{1} \cdot(q-1)^{r} \quad\left(\bmod (q-1)^{r+1}\right) .
$$

In particular, for any infinite set of values of $q$ for which $\# T_{q}$ is a polynomial in $q$ we have that $(q-1)^{r}$ divides $\# T_{q}$ as a polynomial and that $\# T_{q} /\left.(q-1)^{r}\right|_{q=1}=\# T_{1}$.

Proof idea: For each $\ell$, identify $\left(\mathbf{F}_{q}^{\times}\right)^{\ell}$ with the group of invertible diagonal $\ell \times \ell$ matrices. Consider the action of $\left(\mathbf{F}_{q}^{\times}\right)^{m} \times\left(\mathbf{F}_{q}^{\times}\right)^{n}$ on $T_{q}$ given by $(X, Y) \cdot A=X A Y^{-1}$. For any $A \in T_{q}$, let $G$ be the bipartite graph with vertices $v_{1}, \ldots, v_{m}, w_{1}, \ldots, w_{n}$ and an edge $v_{i} w_{j}$ if $A_{i j} \neq 0$. Then $\left(x_{1}, \ldots, x_{m}, y_{1}, \ldots, y_{n}\right) \in\left(\mathbf{F}_{q}^{\times}\right)^{m} \times\left(\mathbf{F}_{q}^{\times}\right)^{n}$ stabilizes $A$ if and only if $x_{i}=y_{j}$ for all edges $v_{i} w_{j}$ of $G$. Thus, the size of the stabilizer of $A$ is $(q-1)^{C(G)}$, where $C(G)$ is the number of connected components of $G$, and the size of the orbit of $A$ is therefore $(q-1)^{m+n-C(G)}$.

Since $A$ has rank $r$, at least $r$ of the $v_{i}$ and $r$ of the $w_{i}$ have positive degree. It follows that $C(G) \leq$ $m+n-r$ with equality if and only if $G$ consists of $r$ disjoint edges, that is, when $G$ is the graph associated to a matrix in $T_{1}$. It follows that the size of each orbit is $(q-1)^{a}$ for some $a \geq r$, and the number of orbits of size $(q-1)^{r}$ is $\# T_{1}$.

Remark 5.2 The technique in the proof of Proposition 5.1 is widely applicable to similar problems. For example, we can use it in the case of symmetric matrices (when $q$ is odd) with rank $2 s$ with zero diagonal. Here, the group $\left(\mathbf{F}_{q}^{\times}\right)^{n}$ of invertible diagonal matrices acts on the set of symmetric matrices by the rule $X \cdot A=X A X$ and we consider the graph $G$ on $n$ vertices $v_{1}, \ldots, v_{n}$ with edge $v_{i} v_{j}$ if and only if $A_{i j} \neq 0$. Proceeding in a similar way it is then possible to conclude that (looking modulo $\left.(q-1)^{s+1}\right)$ we have that symmetric matrices with zero diagonal are a q-analogue of "partial fixed point-free involutions."

\subsection{Polynomiality and a speculation of Kontsevich}

As mentioned in Section 1 , the question of the polynomiality of $\# T_{q}$ is related to a speculation from Kontsevich. We briefly provide some background on Stanley's [Sta2] reformulation of this speculation and on its relation to the polynomiality of $\# T_{q}$.

Let $G$ be an undirected connected graph with edge set $E$, and form the polynomial ring $\mathbf{Z}\left[x_{e} \mid e \in E\right]$. We consider the polynomial $Q_{G}(x)=\sum_{T} \prod_{e \in T} x_{e}$, where the sum is over all spanning trees $T$ of $G$. Let $g_{G}(q)=\#\left\{x \in \mathbf{F}_{q}^{E} \mid Q_{G}(x) \neq 0\right\}$. Kontsevich inquired whether for fixed $G, g_{G}(q)$ is a polynomial function in the parameter $q$.

Let $v_{1}, \ldots, v_{n}$ be the vertices of $G$ and suppose that $v_{n}$ is adjacent to all the other vertices. By the Matrix-Tree Theorem, one may conclude that $g_{G}(q)$ is the number of symmetric matrices in $\mathbf{G L}(n-1, q)$ such that the $(i, j)$-th entry is 0 whenever $i \neq j$ and $v_{i}$ and $v_{j}$ are not connected. Therefore, setting $S_{G}=\left\{(i, j) \mid i \neq j\right.$ and $\left.v_{i} v_{j} \notin E\right\}$ we have $g_{G}(q)=\#\left(T_{q}\left(n \times n, S_{G}, n\right) \cap \operatorname{Sym}(n)\right)$.

Belkale and Brosnan showed in [BB] that Kontsevich's speculation is false by showing that the functions $g_{G}(q)$ are as complicated (in a very precise sense) as the functions counting the number of solutions over $\mathbf{F}_{q}$ of any variety defined over $\mathbf{Z}$. In addition, Stembridge and Schnetz in [Ste1] and [Sch] showed that $g_{G}(q)$ is a polynomial for graphs $G$ with $\leq 12$ and 13 edges respectively, and the latter found six nonisomorphic graphs with 14 edges such that $g_{G}(q)$ is not a polynomial in $q$. Given these results, it becomes 
an interesting problem to determine when $g_{G}(q)$ is a polynomial in $q$. Taken together with Proposition 5.1 . they also suggest the following question:

Question 5.3 For which families of sets $S$ is $\# T_{q}(m \times n, S, r)$ a polynomial in $q$ ?

Note that $\# T_{q}(m \times n, S, r)$ is invariant under permutations of rows and columns. For simplicity, we restrict the question to the case of square matrices. Below, we describe one class of sets $S$ for which the answer is already known by the theory of $q$-rook numbers.

Let $\bar{S}$ denote the complement of the set $S$. We say that $S \subseteq[n] \times[n]$ is a straight shape if its elements form a Young diagram. Thus, to every integer partition $\lambda$ with at most $n$ parts and with largest part at most $n$ (i.e., to each sequence of integers $\left(\lambda_{1}, \lambda_{2}, \ldots, \lambda_{n}\right)$ such that $\left.n \geq \lambda_{1} \geq \lambda_{2} \geq \ldots \geq \lambda_{n} \geq 0\right)$ there is an associated set $S=S_{\lambda}$. We have that $\# S_{\lambda}=\sum \lambda_{i}=|\lambda|$ is the sum of the parts of $\lambda$. Similarly, if $\lambda$ and $\mu$ are partitions such that $S_{\mu} \subseteq S_{\lambda}$ then we say that the set $S_{\lambda} \backslash S_{\mu}$ is a skew shape and we denote it by $S_{\lambda / \mu}$. Next we give three easy facts about straight and skew shapes.

Remark 5.4 (i) Up to a rotation of $[n] \times[n]$, the complement $\overline{S_{\lambda}}$ of the straight shape $S_{\lambda}$ is also a straight shape. However, $\overline{S_{\lambda / \mu}}$ is typically not a skew shape.

(ii) If $(i, j) \in S_{\lambda}$ then the rectangle $\{(s, t) \mid 1 \leq s \leq i, 1 \leq t \leq j\}$ is contained in $S_{\lambda}$. General skew shapes $S_{\lambda / \mu}$ do not have this property.

(iii) If $\lambda=(n, n-1, \ldots, 2,1)$ and $\mu=(n-1, n-2, \ldots, 1,0)$ are so-called "staircase shapes" then $S_{\lambda / \mu}$ is, up to rotation, the set of diagonal entries. Thus the value $\# T_{q}\left(n \times n, S_{\lambda / \mu}, n\right)$ is given in Proposition 2.1 while trivially $T_{q}\left(n \times n, \overline{S_{\lambda / \mu}}, n\right)=\#\{$ invertible diagonal matrices $\}=(q-1)^{n}$.

Given a set $S \subseteq[n] \times[n]$, the $r q$-rook number of Garsia and Remmel $[\overline{\mathrm{GR}}]$ is $R_{r}(S, q)=\sum_{C} q^{\operatorname{inv}(C, S)}$, where the sum is over all rook placements $C \in T_{1}(n \times n, \bar{S}, r)$ of $r$ non-attacking rooks in $S$ and where $\operatorname{inv}(C, S)$ is the number of squares in $S$ not directly above (in the same column) or to the left (in the same row) of any placed rook.

The following result of Haglund shows that when $S=S_{\lambda}$, we have that $T_{q}\left(n \times n, S_{\lambda}, n\right)$ is a polynomial, and in fact is the product of a power of $q-1$ and a polynomial with nonnegative coefficients.

Theorem [Hag, Theorem 1] For straight shapes $S_{\lambda}$, \# $T_{q}\left(n \times n, S_{\lambda}, r\right)=(q-1)^{r} q^{n^{2}-|\lambda|-r} R_{r}\left(\overline{S_{\lambda}}, q^{-1}\right)$.

Question 5.5 The proof of the above theorem relies on Remark 5.4 (ii) and it does not immediately extend to skew shapes $\overline{S_{\lambda / \mu}}$. However, computations using Stembridge's Maple package reduce [Ste2] suggest that when $S$ is a skew shape, \# $T_{q}$ is still a polynomial and that when $S$ is the complement of a skew shape, $\# T_{q}$ is a power of $q-1$ times a polynomial with nonnegative coefficients. Is this true for all skew shapes and their complements?

(Recall that any counter-examples satisfy $n=7$ and $\# S \geq 28$ or $n \geq 8$.)

Question 5.6 Haglund's theorem and the preceding question suggest similarities between \# $T_{q}$ for $S$ and $\bar{S}$ that is reminiscent of the classical reciprocity of rook placements and rook numbers (see [Cho] for a short combinatorial proof). Dworkin [Dwo. Theorem 8.21] gave an analogue of this classical reciprocity for q-rook numbers $R_{r}(S, q)$ when $S=S_{\lambda}$. By Haglund's result, this implies a reciprocity formula relating $T_{q}\left(n \times n, S_{\lambda}, r\right)$ and $T_{q}\left(n \times n, \overline{S_{\lambda}}, r\right)$. Can this reciprocity be extended to skew or other shapes? If so, we could recover the formula for $f_{n, n}$ in Proposition 2.1 from the formula of its complement: $(q-1)^{n}$. 
656 Joel Brewster Lewis, Ricky Ini Liu, Alejandro H. Morales, Greta Panova, Steven V Sam, Yan Zhang

\section{Acknowledgements}

We thank Richard Stanley for suggesting the original problem and Alexander Postnikov for several insights and suggestions. We also thank David Speyer and John Stembridge for helpful discussions and comments, and David Blum for technical support. Steven Sam was supported by an NSF graduate research fellowship and an NDSEG fellowship. Ricky Liu and Yan Zhang were supported by NSF graduate research fellowships.

\section{References}

[Alb] Abraham A. Albert, Symmetric and alternating matrices in an arbitrary field, Trans. Amer. Math. Soc. 43 (1938), 386-436.

[BB] Prakash Belkale and Patrick Brosnan, Matroids, motives and a conjecture of Kontsevich, Duke Math. J. 116 (2003), 147-188, arXiv:math/0012198.

[Ben] Edward A. Bender, On Buckheister's enumeration of $n \times n$ matrices, J. Combin. Theory, Ser. A 17 (1974), 273-274.

[Buc] Philip G. Buckheister, The number of $n \times n$ matrices of rank $r$ and trace $\alpha$ over a finite field, Duke Math. J. 39 (1972), 695-699.

[Cho] Timothy Chow, A short proof of the rook reciprocity theorem, Electron. J. Combin. 3 (1996), no. 1, Research Paper 10.

[Dwo] Morris Dworkin, An interpretation for Garsia and Remmel's $q$-hit numbers, J. Combin. Theory Ser. A 81 (1996), 149-175.

[GR] Adriano M. Garsia and Jeffrey B. Remmel, $q$-counting rook configurations and a formula of Frobenius, $J$. Combin. Theory Ser. A 41 (1986), 246-275.

[Hag] James Haglund, $q$-Rook polynomials and matrices over finite fields, Adv. in Appl. Math. 20 (1998), 450-487, arXiv:math/9706219.

[Jon] Oliver Jones, On the geometry of varities of invertible symmetric and skew-symmetric matrices, Pacific J. Math. 180 (1997), 89-100.

[LLMPSZ] Joel Lewis, Ricky Liu, Alejandro Morales, Greta Panova, Steven Sam and Yan Zhang, Matrices with restricted entries and $q$-analogues of permutations, arXiv:1011.4539v2.

[Mac] Jessie MacWilliams, Orthogonal matrices over finite fields, The American Math. Monthly 76 (1969), no. 2, 152-164.

[Sch] Oliver Schnetz, Quantum Field Theory over $\mathbf{F}_{q}$, arXiv:0909.0905v2.

[Sta1] Richard P. Stanley, Enumerative Combinatorics, vol. I, second edition, available at http: //math.mit. edu/ rstan/ec/ec1/

[Sta2] - Spanning trees and a conjecture of Kontsevich, Ann. Comb. 2 (1998), 351-363.

[Ste1] John Stembridge, Counting points on varieties over finite fields related to a conjecture of Kontsevich, Ann. Comb. 2 (1998), 365-385.

[Ste2] — , reduce Maple package, available at http://www.math.lsa.umich.edu/ jrs/data/ reduce/

[Wan] Zhe-xian Wan, Geometry of Classical Groups over Finite Fields, Studentlitteratur, Lund, Chartwell-Bratt Ltd., Bromley, 1993. 